\title{
Differential outcome expectancies and directed forgetting effects in pigeons
}

\author{
ANGELO SANTI \\ Wilfrid Laurier University, Waterloo, Ontario, Canada
}

\begin{abstract}
Pigeons were trained in a two-choice delayed matching-to-sample task with red and green hues. A brief postsample cue (a vertical or horizontal line) signaled whether the comparison stimuli would be presented or omitted on each trial. Comparison stimuli were always presented following the remember (R) cue, but never following the forget (F) cue or no-cue trials. One group of birds, the differential outcome (DO) group, received reinforcement with a probability of 1.0 for correct responses following one sample stimulus and a probability of 0.2 for correct responses following the other sample stimulus. The nondifferential outcome (NDO) group received reinforcement with a probability of 0.6 for correct responses to either stimulus. The effect of postsample cues was greater for the DO group than for the NDO group. Relative to the NDO group, the DO group displayed higher accuracy on R-cue trials and lower accuracy on F- and no-cue trials. Both tendencies contributed to the enhanced cue effectiveness obtained in the DO group. The results indicate that outcome expectancies are subject to maintenance rehearsal, which comes under the control of postsample $R$ and $F$ cues. They also suggest that maintenance rehearsal may be easier to sustain under DO conditions than under NDO conditions when a memory test is anticipated, but that it may be easier to terminate maintenance rehearsal under DO conditions when a memory test is not anticipated. The results are inconsistent with the assumption that the rehearsal of outcome expectancies is automatic.
\end{abstract}

In a delayed matching-to-sample task, the correct response is to select the comparison stimulus which is the same as the previously presented sample stimulus. As the time between termination of the sample stimulus and presentation of the comparison stimuli increases, accurate performance will increasingly depend upon the animal's ability to maintain information derived from the sample until a correct response can be made. To assess whether pigeons are capable of selectively maintaining information in working memory, researchers have used postsample cues (e.g., a vertical or horizontal line for delayed matching-to-sample of colors) to signal whether comparisons will be omitted or presented on that trial. For example, following a vertical line, comparison stimuli may always be omitted (i.e., a forget, or " $F$ " cue), whereas following a horizontal line, comparison stimuli are always presented (i.e., a remember, or " $R$," cue). By employing a probe-testing procedure in which comparison stimuli are infrequently presented after $F$ cues, it has been shown that $F$ cues control a lower level of matching accuracy than do $R$ cues, which signal presentation of the comparisons (Grant, 1981; Kendrick, Rilling, \& Stonebraker, 1981; Maki \& Hegvik, 1980; Maki, Olson, \& Rego, 1981; Santi \& Savich, 1985). The magnitude of the performance

This research was supported by Grant A6378 from the Natural Sciences and Engineering Research Council of Canada. The author would like to thank Susan Petelka and David Green for their technical assistance. Reprints may be obtained from Angelo Santi, Department of Psychology, Wilfrid Laurier University, Waterloo, Ontario N2L 3C5 Canada. decrement produced by $F$ cues has been shown to be greater as the retention interval is lengthened (Grant, 1981; Maki \& Hegvik, 1980). Furthermore, if the occurrence of the $F$ cue is delayed within the retention interval, the effectiveness of the cue is reduced (Grant, 1981; Stonebraker \& Rilling, 1981). Experiments utilizing two cues have shown that the presentation of an $R$ cue immediately after an $\mathrm{F}$ cue mitigates the decrement in matching accuracy produced by the F cue (Grant, 1981; Stonebraker, Rilling, \& Kendrick, 1981).

Although directed forgetting effects have been most extensively studied in pigeons, they have also been obtained in studies with rats (Grant, 1982) and with squirrel monkeys (Roberts, Mazmanian, \& Kraemer, 1984). Several theoretical interpretations of the directed forgetting effect have been proposed (Grant, 1984, 1986; Maki, 1981; Rilling, Kendrick, \& Stonebraker, 1984), but the one with the greatest degree of support is the rehearsal hypothesis. This hypothesis proposes that the information derived from the sample is actively maintained in working memory by rehearsal. The data obtained from recent studies support the hypothesis that $R$ cues can initiate and maintain this rehearsal process, whereas $F$ cues serve to terminate rehearsal (Grant, 1984; Santi \& Savich, 1985). However, $R$ cues cannot serve as retrieval cues for information that is not being actively rehearsed. Evidence for this comes from Santi and Savich (1985). They found that $\mathbf{R}$ cues failed to control levels of matching accuracy significantly greater than those controlled by $F$ cues if the $R$ cues were delayed until the middle or the end of the retention interval. 
The present research was undertaken to compare directed forgetting effects in a differential-outcome (DO) and a nondifferential-outcome (NDO) condition. A number of studies have demonstrated enhanced acquisition of discrimination and superior memory performance in pigeons when sample stimui are associated with different trial outcomes than when outcomes are nondifferential. The DO effect has been demonstrated with qualitatively different reinforcers (Brodigan \& Peterson, 1976; Edwards, Jagielo, Zentall, \& Hogan, 1982; Honig, Matheson, \& Dodd, 1984; Peterson, Wheeler, \& Armstrong, 1978), reward versus no reward (Peterson, 1984; Peterson \& Trapold, 1980; Peterson, Wheeler, \& Trapold, 1980), biologically neutral outcomes (Fedorchak \& Bolles, 1986), and differential probability of reward (DeLong \& Wasserman, 1981; Santi \& Roberts, 1985a, 1985b). Several alternative explanations of the DO effect have been proposed and tested. The hypothesis with the greatest degree of support maintains that DO expectancies generated by the sample stimuli act as mediating cues and control responding to the comparison stimuli (Edwards et al., 1982; Honig et al., 1984; Peterson, 1984).

Santi and Savich (1985) included a DO and an NDO condition in their study of directed forgetting effects and observed equivalent directed forgetting effects under both conditions. However, the subjects in that study failed to show clear evidence of a DO effect, so the findings with respect to this variable were not particularly informative. It may be that Santi and Savich failed to provide sufficient training with DO conditions. The purpose of the present research, therefore, was to demonstrate that DO effects can be obtained in the context of directed forgetting procedures, and to compare the magnitude of the directed forgetting effects obtained in DO versus NDO treatments.

The enhanced memory performance obtained with DO expectancies suggests that outcome expectancies are relatively easier to rehearse than are memories for the sample stimuli themselves. If this suggestion is correct, it implies that the nature of the memorial representation may influence rehearsal processes. One way of investigating this issue is to examine the magnitude of directed forgetting effects obtained under conditions known to produce different memorial representations. Grant (1984) has argued that there are two types of rehearsal: automatic associative rehearsal and maintenance rehearsal. Maintenance rehearsal is assumed to develop during training and to be under the organism's control; it is the type of rehearsal responsible for directed forgetting effects. Automatic associative rehearsal does not develop during training nor is it controllable by the organism. Grant postulated that this form of rehearsal is responsible for the superior retention of surprising samples relative to expected samples. This hypothesis could be extended to account for the superior retention of DO expectancies. It may be that outcome expectancies provoke automatic associative rehearsal. If this is true, one might expect $F$ cues to be less effective in a DO condition than in an NDO condition because the expectancies would provoke automatic rehearsal on F-cue trials. On the other hand, if $F$ cues are as effective or more effective with DOs than with NDOs, then it would suggest that the superior retention obtained with DO expectancies cannot be explained in terms of automatic associative rehearsal.

Pigeons were trained in a two-choice delayed matchingto-sample task with red and green hues. A brief postsample cue (a vertical or horizontal line) signaled whether the comparison stimuli would be presented or omitted on that trial. Comparison stimuli were always presented on the R-cue trials but were never presented on the F-cue and no-cue trials during training. However, during test sessions, performance was evaluated as a function of the various cue conditions by infrequently presenting comparison stimuli on F-cue and no-cue trials. Probability of reinforcement was the DO (DeLong \& Wasserman, 1981; Santi \& Roberts, 1985a, 1985b). For the DO group, the probabilities of reinforcement were 1.0 and 0.2 , whereas for the NDO group, the probabilities were both 0.6 .

\section{METHOD}

\section{Subjects}

Twelve experimentally naive White Carneaux pigeons, maintained at $80 \% \pm 15 \mathrm{~g}$ of their ad-lib weights and housed individually with constant access to grit and water, served as subjects.

\section{Apparatus}

Four Coulbourn E10-10 modular operant test cages, housed individually in isolation cubicles (Model E10-20), were used. Each cubicle was equipped with a ventilation fan and a baffled air intake and exhaust system. Each test cage was equipped with three horizontally aligned, clear plastic keys behind which projectors could display stimuli (red or green field, vertical or horizontal white line on a black background, or a black dot on a white background) onto a frosted Coulbourn E21-18 rear-projection screen. Directly below the center key was a $5.7 \times 5 \mathrm{~cm}$ opening that provided access to a hopper filled with mixed grain (Coulbourn Model E14-10). All experimental events and response measures were arranged and recorded by a microcomputer system located in an adjacent room.

\section{Procedure}

All birds were hopper-trained and autoshaped to peck the keys. Following this preliminary training, they were given zero-delay matching-to-sample training. Each trial began with a warning signal (a black dot on a white background) presented on the center key. A peck to it resulted in the presentation of either a red or a green sample stimulus on the center key. The sample stimulus was terminated after $5 \mathrm{sec}$ and was followed immediately by presentation of red and green comparison stimuli on the side keys, with positions counterbalanced over trials. A single peck to the comparison stimulus that matched the sample was defined as a correct response and resulted in termination of the comparison stimuli. For the 6 birds in the NDO group, the probability of food reinforcement (3-sec access to mixed grain) was 0.6 following correct responses to either red or green stimuli. For the 6 birds in the DO group, the probability of food reinforcement was 1.0 following correct responses to one sample, and $\mathbf{0 . 2}$ following correct responses to the other sample. For 3 of the DO birds, the probability of reinforcement for correct responses was 1.0 for trials involving a red 
sample; for the other 3 birds, it was 1.0 for trials involving a green sample. For both the DO and the NDO groups, on trials in which reinforcement did not occur, termination of the comparison stimuli was followed by a 3-sec blackout. Following either reinforcement or blackout, an intertrial interval of $10 \mathrm{sec}$, spent in darkness, occurred. Each session consisted of 60 trials (15 individually randomized blocks of the four different stimulus configurations). The birds in both the DO and the NDO groups were given 25 sessions of training with a 0 -sec delay. The delay was then increased to $2 \mathrm{sec}$ and the birds were given 20 sessions of training. The performance of 1 of the NDO birds fell to chance levels during this phase and it was dropped from the study.

In the next phase of training, termination of the sample stimulus was followed by the presentation of a 1-sec postsample cue. The postsample cue was a vertical line, a horizontal line, or no cue (i.e., the absence of any signal for $1 \mathrm{sec}$ ). For 5 birds ( 3 DO and 2 NDO), the vertical line signaled the occurrence of comparison stimuli ( $R$ cue), whereas the horizontal line signaled their nonoccurrence (F cue). For the other 6 birds ( $3 \mathrm{DO}, 3 \mathrm{NDO}$ ), the cue functions were reversed. For all birds, the no-cue condition signaled the nonoccurrence of comparison stimuli. Red and green comparison stimuli were presented on the side keys at the end of the delay interval only if the sample had been followed by an $R$ cue. The delay interval as measured from the offset of the postsample cue was $1 \mathrm{sec}$ for the first $\mathbf{2 0}$ sessions and was then incremented up to $7 \mathrm{sec}$ over the next 75 sessions. There were 20 sessions with a 3 -sec delay, 15 sessions with a 4-sec delay, 20 sessions with a 5-sec delay, and 20 sessions with a 7-sec delay. Further training was given at shorter and longer delays for $\mathbf{5 0}$ additional sessions but these data will not be reported. The performance of 1 additional NDO bird dropped to very low levels during this phase and it was terminated from the study. Dropping 2 birds from the NDO group because of very low levels of accuracy works against obtaining differences between the DO and the NDO groups.

Immediately prior to testing for effectiveness of the postsample cues, the delay interval was shortened to $0 \mathrm{sec}$ for seven sessions. Over the last three sessions of this phase, the 6 DO birds averaged $97.2 \%$ correct and the 4 NDO birds averaged $96.7 \%$ correct. Each test session consisted of 60 regular trials, which were the same as during baseline training, and an additional 12 probe trials. The 12 probe trials in each test session consisted of four $R$-cue, four F-cue, and four no-cue trials. A single probe trial occurred randomly within each block of five regular trials. On probe trials, comparison stimuli were always presented, regardless of the nature of the cue presented on that trial, and correct responses were reinforced according to the contingencies previously described. The delay-interval duration was varied between sessions during testing in 10 blocks of 3 days each. There were three delay intervals tested: 1,5 , and $10 \mathrm{sec}$. A total of $\mathbf{3 0}$ test sessions was given.

\section{RESULTS}

The mean percentage of correct matching responses obtained during the training sessions prior to testing were collapsed into blocks of five sessions and are shown in Figure 1. Both the DO and the NDO groups acquired the delayed-matching task at equivalent rates. However, when the delay interval was increased to $2 \mathrm{sec}$, the DO group had higher levels of accuracy than did the NDO group $\left[F(1,10)=5.35, M S_{\mathrm{e}}=397.55, p<.05\right]$. The introduction of the directed forgetting procedure in the next phase of training eliminated the performance difference between the DO and NDO groups. This occurred as a result of a decrease in accuracy following the introduction of the R and F cues for the DO group. Over the re- maining blocks of sessions, the delay interval was incremented from 1 to $7 \mathrm{sec}$. As can be seen in Figure 1, incrementing the delay interval reestablished the performance difference in favor of the DO group. This difference was statistically significant at the 7-sec delay $\left[F(1,9)=6.42, M S_{e}=387.72, p<.05\right]$.

The mean percentage of correct matching responses obtained during cue-testing sessions are presented in Figure 2. An analysis of variance performed on the test data included type of cue and delay interval as withinsubjects factors, and DO condition as a between-subjects factor. The effect of cue type $\left[F(2,16)=34.80, M S_{\mathrm{e}}=\right.$ $99.49, p<.001]$ and delay interval $[F(2,16)=44.02$, $\left.M S_{\mathrm{e}}=44.87, p<.001\right]$ were statistically significant . In addition, DO condition interacted significantly with both cue type $\left[F(2,16)=9.46, M S_{\mathrm{e}}=99.49, p<.01\right]$ and delay interval $\left[F(2,16)=3.68, M S_{\mathrm{e}}=44.87, p<\right.$ .05]. The interaction of group and cue type was due to the cuing effect's being much stronger for the DO group $[F(2,16)=50.34]$ than for the NDO group $[F(2,16)=$ 3.32]. In both groups, there was no difference in accuracy between F-cue and no-cue trials $[t(16)=1.20$ for the DO group; $t(16)<1$ for the NDO group]. However, performance on $\mathbf{R}$-cue trials was significantly greater than the averaged performance on F-cue and no-cue trials for both the DO $[t(16)=9.97, p<.001]$ and the NDO conditions $[t(16)=2.55, p<.05]$. In neither group was there any indication of accelerated forgetting in the F-cue or no-cue condition as the delay interval was lengthened. However, increasing the delay interval had a greater effect in the NDO condition $[F(2,16)=28.86]$ than in the DO condition $[F(2,16)=16.33]$. Additional analyses indicated that accuracy on R-cue trials was equivalent for the DO and NDO groups at the two shorter delays, but there was a tendency for accuracy to be higher in the DO group on R-cue trials at the longest delay $[F(1,8)=4.99$, $p=.056]$. The averaged accuracy on F-cue and no-cue trials at the 1-sec delay was lower in the DO group than in the NDO group; however, this difference failed to achieve statistical significance $[F(1,8)=3.59, p>.094]$. These additional analyses show that the significantly greater cuing effect in the DO group cannot be attributed solely to higher accuracy on R-cue trials or to lower accuracy on F-and no-cue trials in that group. Both tendencies contributed to the enhanced cue effectiveness obtained in the DO group.

An analysis of performance on high- and low-probabilityof-reinforcement trials was undertaken for the DO group. These data are presented in Table 1 . There was a significant effect of type of cue $\left[F(2,10)=43.56, M S_{e}=\right.$ $229.95, p<.001]$, and a significant interaction of type of cue and probability of reinforcement $[F(2,10)=5.64$, $\left.M S_{\mathrm{e}}=218.29, p<.05\right]$. A simple main-effects analysis showed that accuracy on R-cue trials was significantly greater when the probability of reinforcement was high than when it was low $[F(1,5)=17.74]$. However, accuracy on high- and low-probability-of-reinforcement trials did not differ in the F- or no-cue condition (both $F \mathrm{~s}<1$ ). 


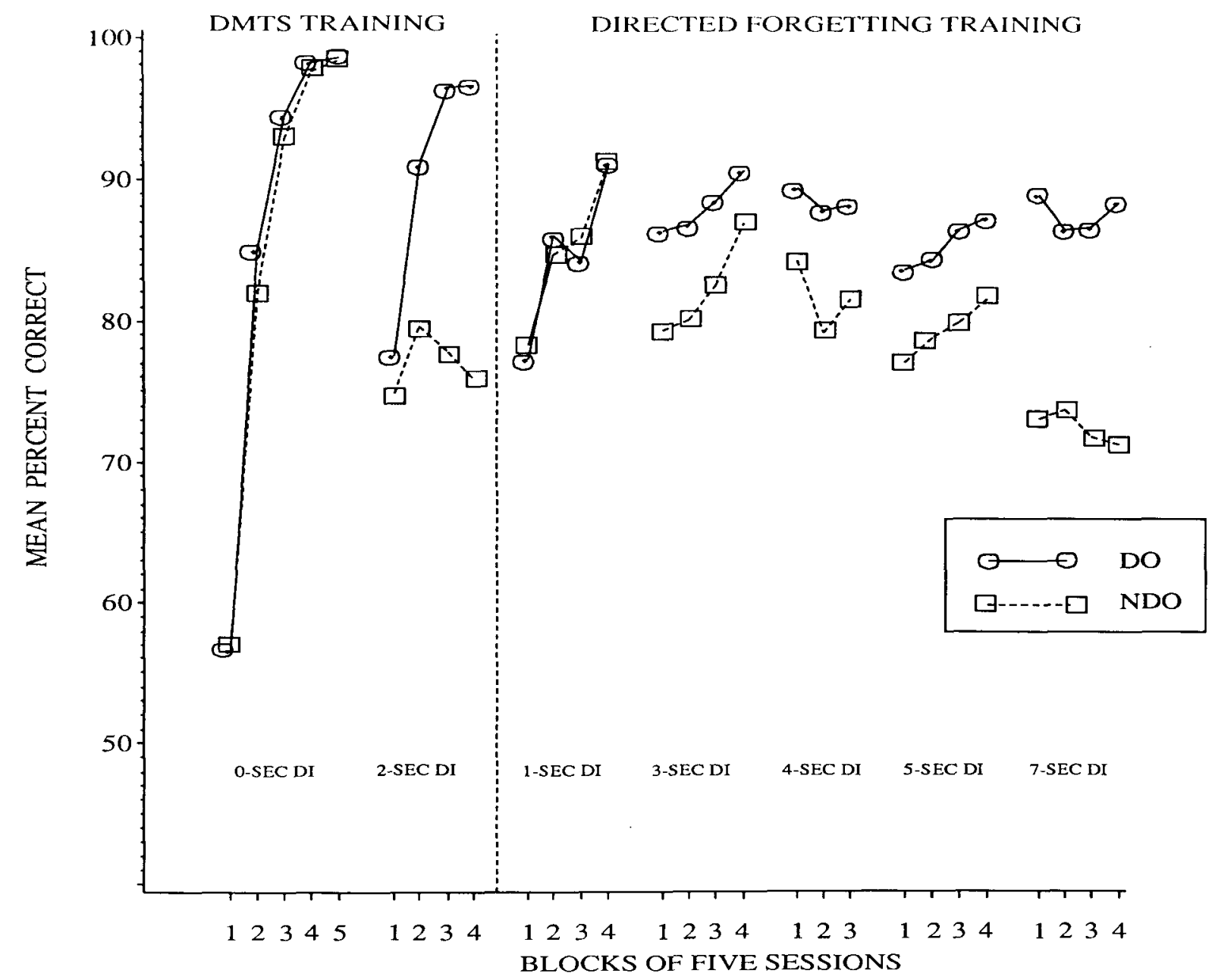

Figure 1. Mean percentage of correct responses during delayed matching-to-sample (DMTS) and directed forgetting training for the differential outcome (DO) and nondifferential outcome (NDO) groups as a function of various delay interval (DI) values.

Most importantly, although the effect of postsample cues was strongest on high-probability-of-reinforcement trials $[F(2,10)=32.92]$, there was a significant effect of cuing on low-probability-of-reinforcement trials as well $[F(2,10)=12.38]$. Finally, although accuracy on both high- and low-probability-of-reinforcement trials decreased as delay-interval duration increased $[F(2,10)=15.39$, $M S_{\mathrm{e}}=95.23, p<.001 ;$ not shown in Table 1], there was no interaction of reinforcement probability and delay interval $(F<1)$.

\section{DISCUSSION}

The present experiment was successful in obtaining a DO effect with differential probability of reinforcement in a directed-forgetting procedure. This study helps to extend the range of conditions under which DO effects can be observed (DeLong \& Wasserman, 1981; Santi \& Roberts, 1985a, 1985b). The failure of Santi and Savich (1985) to obtain this effect cannot be attributed to their use of a directed forgetting procedure; they probably did not provide sufficient training with DO conditions. In the present experiment, for both DO and NDO groups, matching accuracy of F-cue and no-cue trials was equivalent and inferior to that on R-cue trials. This result replicates those reported by Santi and Savich. It shows that the omission of comparison stimuli on no-cue trials resulted in the no-cue condition functioning as an implicit $F$ cue. This finding supports Grant's (1981) suggestion that previous training is a critical factor in determining whether the nocue condition will act as an implicit $R$ or $F$ cue.

The most important result obtained in the present study was the enhanced effectiveness of postsample cues when DO expectancies were available. The enhanced memory performance shown by subjects trained with DOs both in the present study and in several previous studies (DeLong \& Wasserman, 1981; Edwards et al., 1982; Peterson et al., 1980) has generally been assumed to be due to outcome expectancies being easier to rehearse than is information about the sample itself. If this assumption is taken to mean that the rehearsal of outcome expectancies is in some sense automatic (Grant, 1984), then one would ex- 

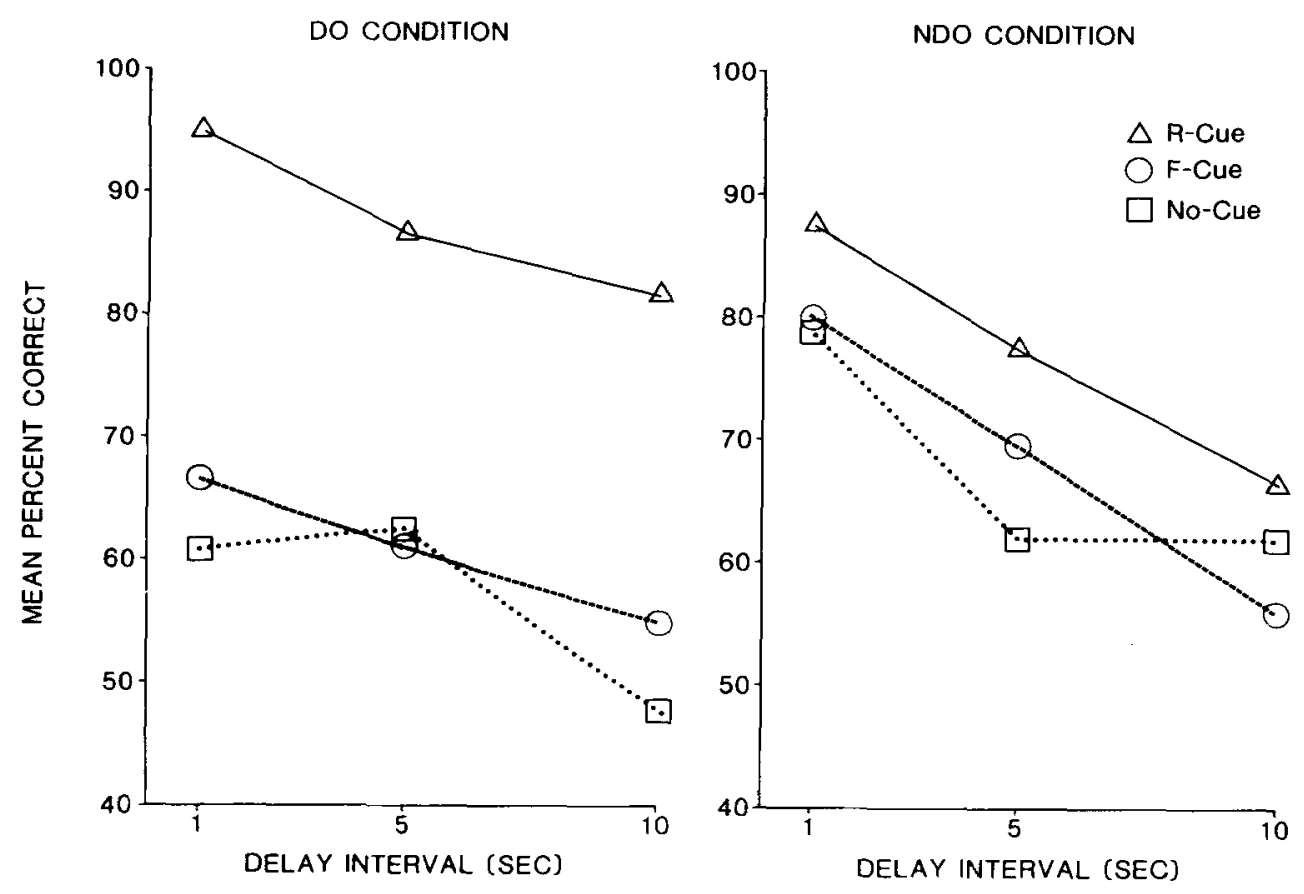

Figure 2. Mean percentage of correct responses during testing as a function of delay interval, type of cue ( $R$ cue, $F$ cue, or no cue), and differential outcome group (DO vs. NDO).

pect $F$ cues to be less effective in a DO condition than in an NDO condition. The present results are inconsistent with the assumption that the rehearsal of outcome expectancies is automatic. Outcome expectancies are subject to maintenance rehearsal, and this form of rehearsal clearly comes under the control of postsample $R$ and $F$ cues. The data indicate that the enhanced effectiveness of postsample $\mathbf{R}$ and $\mathrm{F}$ cues in the DO group relative to the NDO group cannot be attributed exclusively either to greater accuracy on R-cue trials in the DO group or to lower accuracy on F- and no-cue trials. It appears that both tendencies were responsible for the enhanced cue effectiveness shown in the DO group. Although the data do not permit a strong conclusion to be reached at this time, they suggest that maintenance rehearsal may be easier to sustain under DO conditions than under NDO conditions when a memory test is anticipated, but that it may be easier to terminate maintenance rehearsal under DO conditions when a memory test is not anticipated.

The analysis of performance on high- and lowprobability-of-reinforcement trials for the DO group showed that the directed-forgetting effect occurred on both

Table 1

Mean Percentage of Correct Matching on High- and Low-Probabilityof-Reinforcement Trials in the Differential Outcome Group

\begin{tabular}{cccc}
\hline \multirow{2}{*}{$\begin{array}{c}\text { Probability } \\
\text { of Reinforcement }\end{array}$} & R Cue & F Cue & No Cue \\
\hline High & 95.6 & 59.4 & 54.2 \\
Low & 80.0 & 62.8 & 60.0 \\
\hline
\end{tabular}

Note $-\mathrm{R}$ cue $=$ remember cue; $\mathrm{F}$ cue $=$ forget cue. types of trials and that there did not appear to be a strong bias to select the comparison associated with the low probability of reinforcement on F- and no-cue trials. One might anticipate such a bias if the birds generalized between an expectancy of no reinforcement on F- and no-cue trials, and an expectancy of a low probability of reinforcement. However, on high-probability-of-reinforcement trials, the birds tended to choose the stimulus associated with the 1.0 reinforcement probability at a level slightly above chance following the $F$ cue or no cue, whereas on lowprobability trials, they tended to choose the stimulus associated with the 0.2 reinforcement probability at a level slightly above chance following the same cues. Consequently, it is not the case that the reduced accuracy on F- and no-cue trials for the DO group was due to a tendency to choose the stimulus associated with the 0.2 reinforcement probability. In addition, these data reveal that accuracy declined similarly on high- and low-probabilityof-reinforcement trials as the delay interval was lengthened. Santi and Roberts (1985b) reported a similar result. It appears that the use of differential reinforcement probabilities does not result in a strong bias to select the comparison associated with either the high or the low reinforcement probability as the relevant trial information is lost.

Further research will assess whether the differential directed forgetting effects observed in the DO and NDO groups can also be obtained when qualitatively different reinforcers are used as the DOs. It may be the case that the differences observed in the magnitude of directed forgetting effects was due to differences in the discrimina- 
bility of the memorial representations. That is, the control exerted by $R$ and $F$ cues may be stronger when the memory codes are highly discriminable than when they are less discriminable. Examining directed forgetting effects as a function of variations in the discriminability of sample stimuli would provide evidence with respect to this hypothesis.

In summary, the present results have demonstrated that DO effects can be obtained following training with a directed forgetting procedure. In addition, they indicate that the rehearsal of DO expectancies is not automatic, but rather is subject to control by postsample $R$ and F cues.

\section{REFERENCES}

Brodigan, D. L., \& Peterson, G. B. (1976). Two-choice conditional discrimination performance of pigeons as a function of reward expectancy, prechoice delay, and domesticity. Animal Learning \& Behavior, 4, 121-124.

DeLong, R. E., \& Wasserman, E. A. (1981). Effects of differential reinforcement expectancies on successive matching-to-sample performance in pigeons. Journal of Experimental Psychology: Animal Behavior Processes, 7, 394-412.

Edwards, C. A., Jagielo, J. A., Zentall, T. R, \& Hogan, D. E. (1982). Acquired equivalence and distinctiveness in matching to sample by pigeons: Mediation by reinforcer-specific expectancies. Journal of Experimental Psychology: Animal Behavior Processes, 8, 244-259.

FedorChAK, P. M., \& Bolles, R. C. (1986). Differential outcome effect using a biologically neutral outcome difference. Journal of Experimental Psychology: Animal Behavior Processes, 12, 125-130.

GraNT, D. S. (1981). Stimulus control of information processing in pigeon short-term memory. Learning \& Motivation, 12, 19-39.

GraNT, D. S. (1982). Stimulus control of information processing in rat short-term memory. Journal of Experimental Psychology: Animal Behavior Processes, 8, 154-164.

GRANT, D. S. (1984). Directed forgetting and intratrial interference in pigeon delayed matching. Canadian Journal of Psychology, 38, 166-177.

GRANT, D. S. (1986). Establishing a forget cue in pigeons using the intratrial interference procedure. Animal Learning \& Behavior, 14, 267-275.

Honig, W. K., Matheson, W. R., \& Dodd, P. W. D. (1984). Outcome expectancies as mediators for discriminative responding. Canadian Joumal of Psychology, 38, 196-217.

Kendrick, D. F., Rilling, M., Stonebraker, T. B. (1981). Stimu- lus control of delayed matching in pigeons: Directed forgetting. Journal of the Experimental Analysis of Behavior, 36, 241-251.

MAKI, W. S. (1981). Directed forgetting in animals. In N. E. Spear \& R. R. Miller (Eds.), Information processing in animals: Memory mechanisms (pp. 199-226). Hillsdale, NJ: Erlbaum.

MaKI, W. S., \& Hegvik, D. K. (1980). Directed forgetting in pigeons. Animal Learning \& Behavior, 8, 567-574.

MaKi, W. S., Olson, D., \& Rego, S. (1981). Directed forgetting in pigeons: Analysis of cue functions. Animal Learning \& Behavior, 9 , 189-195.

Peterson, G. B. (1984). How expectancies guide behavior. In H. L. Roitblat, T. G. Bever, \& H. S. Terrace (Eds.), Animal cognition (pp. 135-148). Hillsdale, NJ: Erlbaum.

Peterson, G. B., \& Trapold, M. A. (1980). Effects of altering outcome expectancies on pigeons' delayed conditional discrimination performance. Learning \& Motivation, 11, 267-288.

Peterson, G. B., Wheeler, R. L., \& Armstrong, G. D. (1978). Expectancies as mediators in the differential-reward conditional discrimination performance of pigeons. Animal Learning \& Behavior, 6, 279-285.

Peterson, G. B., Wheeler, R. L., \& Trapold, M. A. (1980). Enhancement of pigeons' conditional discrimination performance by expectancies of reinforcement and nonreinforcement. Animal Learning \& Behavior, 8, 22-30.

Rilling, M., Kendrick, D. F., \& Stonebraker, T. B. (1984). Directed forgetting in context. In G. H. Bower (Ed.), The psychology of learning and motivation: Advances in research and theory (Vol. 18, pp. 175-198). New York: Academic Press.

Roberts, W. A., Mazmanian, D. S., \& Kraemer, P. J. (1984). Directed forgetting in monkeys. Animal Learning \& Behavior, 12, 29-40.

Santi, A., \& Roberts, W. A. (1985a). Prospective representation: The effects of varied mapping of sample stimuli to comparison stimuli and differential trial outcomes on pigeons' working memory. Animal Learning \& Behavior, 13, 103-108.

SANTI, A., \& RoBERTS, W. A. (1985b). Reinforcement expectancy and trial spacing effects in delayed matching to sample by pigeons. Animal Learning \& Behavior, 13, 274-284.

SanTI, A., \& SA VICH, J. (1985). Directed forgetting effects in pigeons: Remember cues initiate rehearsal. Animal Learning \& Behavior, 13, 365-369.

Stonebraker, T. B., \& Rilling, M. (1981). Control of delayed matching-to-sample performance using directed forgetting techniques. Animal Learning \& Behavior, 9, 196-201.

Stonebraker, T. B., Rilling, M., \& Kendrick, D. F. (1981). Time dependent effects of double-cuing in directed forgetting. Animal Learning \& Behavior, 9, 385-394.

(Manuscript received July 19, 1988;

revision accepted for publication October 18,1988 .) 